\title{
Review Article \\ Restoration Methods of Respiratory Function for Spinal Cord Injury
}

\author{
X. Y. Gu $\mathbb{D}^{1},{ }^{1}$ S. Ren $\mathbb{D}^{2,3}{ }^{2,3}$ Y. Shi $\mathbb{D}^{2},{ }^{2}$ N. Wang $\mathbb{D}^{4},{ }^{4}$ Z. H. Tong ${ }^{\circ},{ }^{5}$ and M. L. Cai ${ }^{2}$ \\ ${ }^{1}$ The School of Biology and Medical Engineering, Beihang University, Beijing 100191, China \\ ${ }^{2}$ The School of Automation Science and Electrical Engineering, Beihang University, Beijing 100191, China \\ ${ }^{3}$ The State Key Laboratory of Fluid Power and Mechatronic Systems, Zhejiang University, Hangzhou 310027, China \\ ${ }^{4}$ The Engineering Training Center, Beihang University, Beijing 100191, China \\ ${ }^{5}$ The Department of Respiratory Medicine, Beijing Chaoyang Hospital, Beijing 100020, China
}

Correspondence should be addressed to S. Ren; renshuai@buaa.edu.cn and Y. Shi; shiyan@buaa.edu.cn

Received 29 May 2020; Revised 27 June 2020; Accepted 8 July 2020; Published 29 July 2020

Guest Editor: Hang Su

Copyright ( $\odot 2020$ X. Y. Gu et al. This is an open access article distributed under the Creative Commons Attribution License, which permits unrestricted use, distribution, and reproduction in any medium, provided the original work is properly cited.

Respiratory dysfunction caused by high spinal cord injury is fatal damage. Three treatment methods commonly used in the clinic, diaphragm pacing, mechanical ventilation, and respiratory muscle training, were chosen to explain the respiratory function reconstruction of spinal cord injury. The characteristics, research status, advantages, and disadvantages of these three treatment methods are reviewed. Diaphragm pacing technology has attracted much attention due to its price-friendly, efficient, and closer to physiological respiration. Therefore, the emphasis is on describing the characteristics of the stimulation waveform of diaphragm pacing and the mathematical correspondence between stimulation parameters (pulse interval, inspiratory time, etc.) and tidal volume. Meanwhile, it also briefly introduces that for patients with SCI with poor diaphragm pacing, intercostal muscle pacing can be used as the second option to restore respiratory function. Also, the development of electronic technology has promoted the emergence of closed-loop diaphragm pacing technology. Finally, we propose that the method of respiratory function reconstruction after spinal cord injury should pay more attention to physiology and the safety of surgery.

\section{Introduction}

Recently, with the advance of transportation and construction industry, the number of spinal cord injury (SCI) patients caused by traffic accidents and falls has been increasing year by year. According to the survey, the global incidence of traumatic spinal cord injury (TSCI) is almost $10.5 / 100,000$ [1]. SCI in China has exceeded 100,0000 cases in 2012 and increases by 10,000 to 60,000 cases each year [2]. High segment and severe SCI, especially for patients with complete high cervical SCI, will lead to respiratory dysfunction and high mortality [2]. In the critical condition, about $20 \%$ of these patients need to be treated with tracheotomy and mechanical ventilation. After treatment, there are still 5\% of patients with acute SCI depending on longterm ventilation support with a ventilator to sustain life [3]. It may cause the patients speech difficulties, inconvenience in social activities, decubitus ulcers, and lung infection, bringing them both psychological and physical pressure [4].

In the past several decades, functional electrical stimulation, using electricity to stimulate the motor nerve to cause muscles contraction, has been successfully used in the recovery of motor function in SCI. Based on this principle, the implantable diaphragmatic pacing can restore spontaneous respiration in high cervical SCI patients [5]. Applying abdominal functional electrical stimulation can increase the tidal volume of respiration in patients with SCI [6]. Furthermore, Electrical stimulation of intercostal nerve roots $(\mathrm{T} 2,3)$ produces higher inspiratory capacity $[7,8]$. Besides, there are several other ways to reconstruct the respiratory function after SCI, such as the nerve and olfactory cell transplantation $[9,10]$.

As mentioned previously, the ways to restore the patients' respiratory function with SCI are varied. It is 
inappropriate for us to describe all the methods in detail. Hence, we choose three mature clinical treatments with the help of medical devices, including mechanical ventilation, functional electrical stimulation, and respiratory muscle training for analysis. By comparing the effectiveness, advantages, and disadvantages of these three methods to patients' treatment, we focus on the electrical stimulation and briefly describe the clinical application of the other two techniques. Finally, the future development of respiratory function reconstruction methods for SCI is briefly introduced. This review is useful for engineering technicians and medical staff engaged in respiratory therapy. For medical staff, they can quickly understand the key technologies and indications of these treatments to choose the appropriate treatment for the patient. The engineering staff would propose improvements to the technical deficiencies of existing treatment equipment to promote the development of medical devices related to respiratory therapy.

\section{Respiratory Mechanism and Spinal Cord Injury}

The respiration motion, consisting of inhalation and exhalation, can be seen as the process of gas exchange between the body and the external environment. It is always a selfcontrol and unconscious movement [11], which is controlled by a combination of respiratory muscles, such as the diaphragm, intercostal muscles, and scalenus muscles. The contraction of diaphragm, intercostals, and scalenus muscles in inhalation leads to the decrease in chest pressure. Therefore, the increase of lung volume and the reductions of pressure in lung make the gas from the outside enter the body. During exhalation, the movement of the respiratory muscles and changes of the air pressure in the chest and lungs are contrary to the inhalation process.

In the respiratory muscles, the diaphragm separating the chest and the abdominal cavity is the primary power pump source of ventilation function [12]. The volume of ventilation produced by diaphragm contraction in rest respiration accounts for $60 \%-80 \%$ of the total amount of ventilation [12].

The spinal cord can be seen as the bridge between the brain and the peripheral nerves. Its function is to transmit the motor and sensory information to brain and organs of the body [2]. As shown in Figure 1, the spinal cord in the spine made up of 26 segment vertebras controls different physiological (sensation and motor) functions of the body $[3,13]$.

High spinal cord injury (cervical and upper thoracic SCI) is a fatal injury that results in respiratory dysfunction, expectoration weakness, and even without autonomous breathing. That is because high SCI damage the neurons that dominate the contraction of the central respiratory muscles [3]. Figure 2 simply illustrates that the respiratory rhythm arises from the medulla oblongata and its relationship to the spinal cord. The axons of the respiratory neurons project down into the spinal cord, causing the motor neurons of the inspiratory muscles (diaphragms and intercostal muscles) to excite. If the injury occurs above T6, the medullary centre

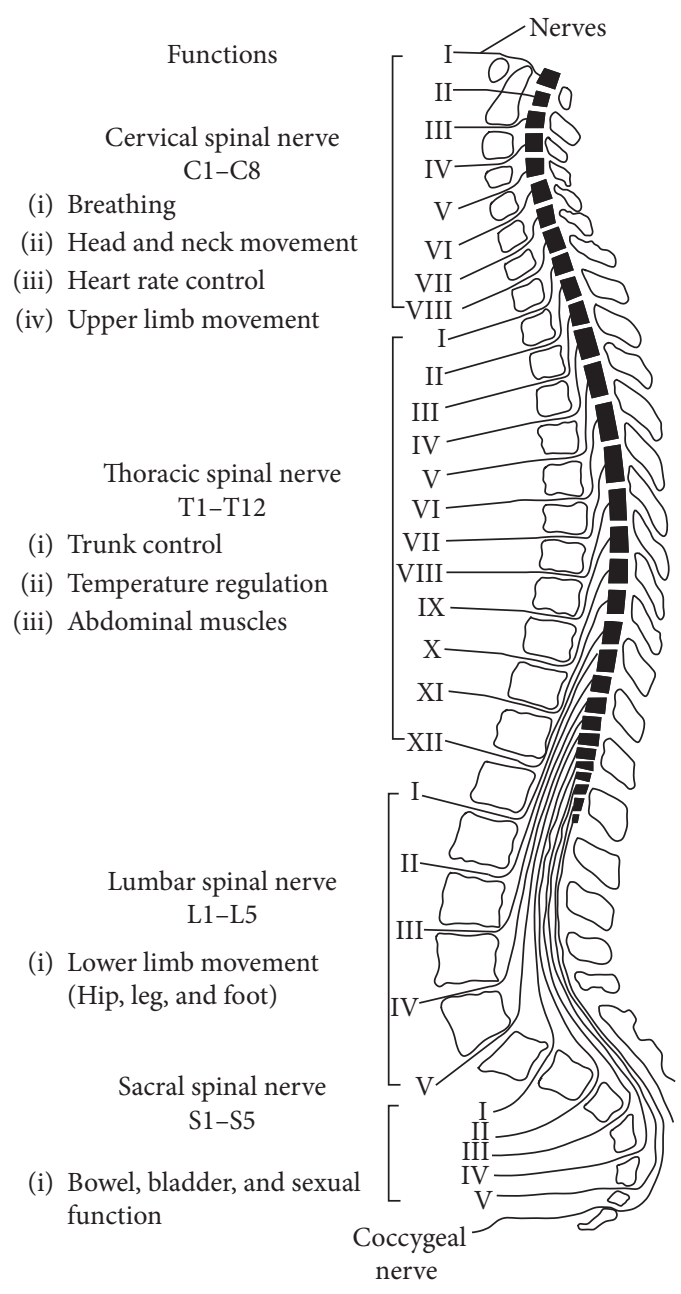

FIgURE 1: The structure of the spine and its corresponding physiological function [13]. The spine is composed of 26 segments of vertebras, which can be divided into the cervical spine (7), thoracic spine (12), lumbar spine (5), sacroiliac spine, and coccyx. A spinal canal is formed inside the spine from the top to bottom with a spinal cord inside. Spinal cords in different segments control different physiological functions (breathing, movement, defecation function, etc.) of the human body.

and the respiratory muscles will be separated to different degrees, causing respiratory dysfunction. Especially for damages above the $\mathrm{C} 2$ level, the medulla oblongata will completely lose regulation of the respiratory muscles.

It is reported that the incidence of respiratory complications, including ventilatory failure and respiratory infection, caused by high SCI can be as high as $81.3 \%[14,15]$. More significantly, higher (C1-C4) and more severe spinal cord injuries will lead to more severe and long-lasting respiratory complications.

\section{Electrical Stimulation Therapy}

In recent decades, functional electrical stimulation has achieved a specific clinical effect in the recovery of movement, respiration, urination, and other functions of patients with SCI. It is reported that the diaphragm has been 


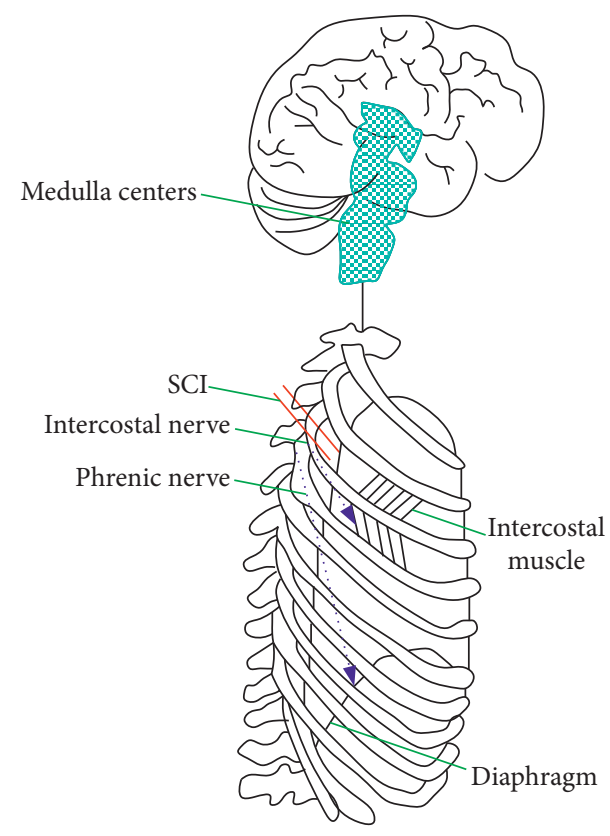

FIGURE 2: The relationship between SCI and respiratory dysfunction. There is a primary centre in the medulla that produces rhythmic breathing. SCI will cause respiratory muscles to escape the control of the medulla centre.

activated by electrical stimulation technology for more than 200 years [16].

3.1. Diaphragm Pacemaker. Diaphragmatic pacing (DP), also known as phrenic nerve stimulation (PNS), is based on the principle of stimulating the phrenic nerve with an electric pulse, thus causing the contraction of the diaphragm to simulate the respiratory movement of the human physiological mode [17]. In the 1960s, the development of electrical stimulation by radio-frequency changed the conventional PNS system. In 1972, Glenn et al. used DP for the first time to improve the respiratory function of patients with high cervical spinal cord injury and achieved success [18].

Moreover, DP technology was proved to be a safe and reliable operation. Glenn being the first doctor to use DP, reported the follow-up results of 77 patients in 1985 [19]. DP was used for up to 10-16 years in these 77 patients. Kong et al. introduced that, in 19 patients who used phrenic stimulation in Australia, the average duration of continuous pacing reached 13 years [20]. There are also many cases that have been used for more than 20 years [21]. At present, DP technology has been recognized in clinical. According to some studies, more than 2000 patients in the world have used DP to recover the SCI that cannot breathe autonomously $[22,23]$. These cases fully prove the effectiveness and safety of DP.

Figure 3(a) is the operating principle of diaphragm pacemaker (DP) containing implanted components (electrodes, connector, and radiofrequency receiver) and exterior components (antenna and stimulus transmitter) [24]. Figure $3(\mathrm{~b})$ is a physical diagram of the implantation of electrodes and receivers for diaphragm pacemaker surgery. The two electrodes connected to the radiofrequency receiver are placed on the phrenic nerves. There is no electric source in vivo, and the external stimulus transmitter based on the principle of electromagnetic induction provides parameter commands (waveform, pulse width, and frequency) and electric energy for the internal device. During stimulation, the power and stimulus parameter instruction is sent to the radiofrequency receiver by the stimulus transmitter. According to the received data, the radiofrequency receiver transmits the electric pulse to the phrenic nerve through the electrode in an alternating manner.

The working process of the DP and the relationship between the various components can be summarised in the flowchart shown in Figure 4. It can be seen more intuitively from the workflow diagram that the DP does not monitor the ventilation effect during the work process, which may be dangerous without the care of medical staff.

Currently, three kinds of DP manufactured by Avery company in the United States, Atrotech company in Finland, and Medimplant company in Australia, respectively, are available in the market $[4,26]$. The stimulation effect of them is the same, and the main difference (see Table 1) is the number of the electrode and the electrode implantation. Among the three types of DP, Avery is the first company to develop this product widely used in the world. Compared with the bipolar electrode, the monopolar electrode with more advantages is suitable for most patients. However, for patients with heart pacemaker, to prevent the interference of current, the only bipolar electrode can be selected. Atrotech and Medimplant use quadrupole electrodes to reduce the stimulation frequency and effectively reduce diaphragm fatigue.

3.1.1. Indication of $D P$. The main indications of DP are sleep apnea syndrome, brain stem injury-induced respiratory failure, and loss of spontaneous respiration due to SCI above C3 [27]. Among these injuries, high SCI is the best indication for DP [28]. However, not all respiratory dysfunction caused by high SCI is suitable for treatment with DP. Whether the structure and function of the phrenic nerve and the phrenic muscle are complete is the fundamental premise for performing DP. Therefore, it is necessary to evaluate it in detail before surgery $[29,30]$. The evaluation methods of diaphragm function mainly include the phrenic nerve conduction velocity $(7.5 \sim 9 \mathrm{~ms})$, the change in transdiaphragm pressure (unilateral pacing: $10 \mathrm{~cm} \mathrm{H}_{2} \mathrm{O}$ ), and the observation of diaphragm activity under fluoroscopy [31-33], which are as shown in Table 2. Fluoroscopy is the most reliable method to observe diaphragm activity among these methods. X-ray fluoroscopy was used to observe the degree of phrenic muscle descent when the phrenic nerve was electrically stimulated. If the descent was more than $5 \mathrm{~cm}$, the success rate of pacing was high.

In addition, the pacing response of unilateral diaphragm may not hinder the implementation of diaphragm pacing. On the contrary, unilateral diaphragm pacing can achieve good pacing effect $[30,34]$. Oldenburg $\mathrm{O}$. verified that the 


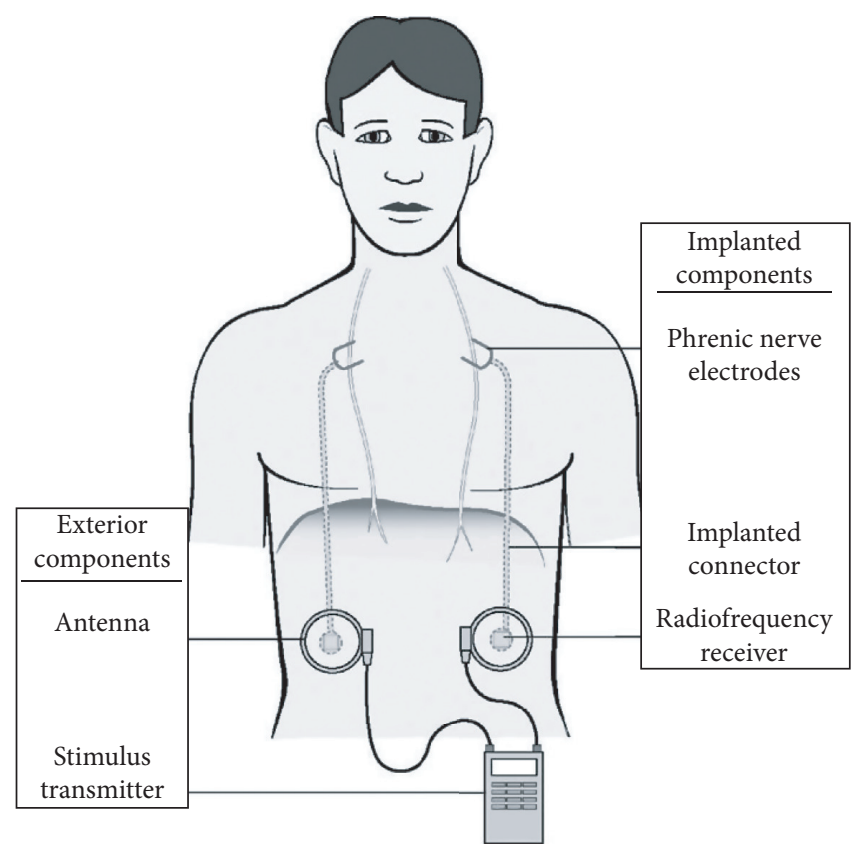

(a)
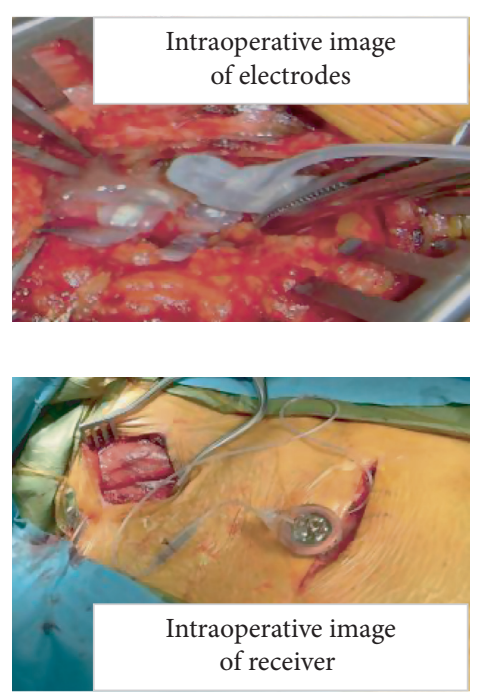

(b)

Figure 3: General view of the diaphragmatic pacing system $[24,25]$. (a) It contains the implanted part and exterior part, and the communication method between them is wireless transmission. The external controller regulates phrenic nerve stimulation parameters (waveform, frequency, respiratory cycle, etc.). The internal part is responsible for transmitting stimulation parameters to the phrenic nerve to control diaphragm contraction. (b) Implantation of diaphragm pacemaker components.

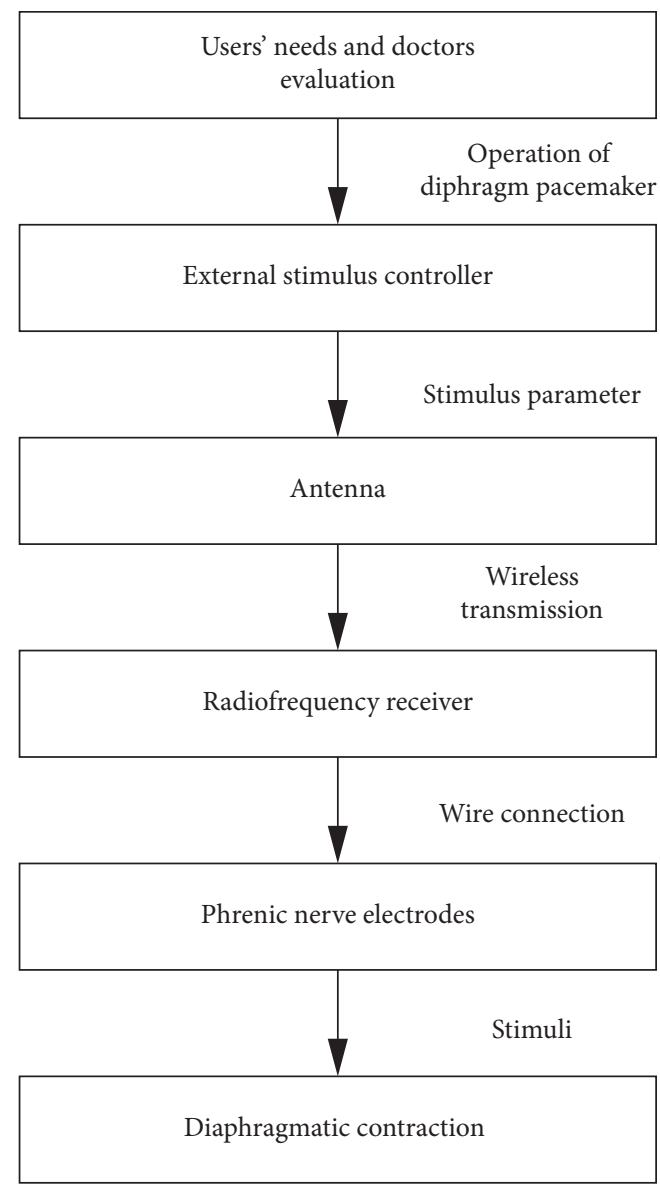

FIGURE 4: DP working flowchart. It is drawn according to the principles introduced earlier. tidal volume generated by unilateral phrenic nerve stimulation was maintained at $700-1000 \mathrm{ml}$ at $12 / \mathrm{min}, 16 / \mathrm{min}$, and $18 / \mathrm{min}$, which fully meets the physiological needs of human respiration [30].

3.1.2. Parameter of DP. The stimulation waveform of phrenic nerve is displayed in Figure 5 [35]. T1 and T2 represent the time of the respiratory cycle and inspiration respectively, so the expiratory time equals to the difference between T1 and T2. Each blue vertical line in Figure 5 denotes a stimulus pulse, and its pulse width is Ti. In the inspiratory phase (T2), the pulses (ITH is the minimum stimulation threshold, and ITV is the maximum stimulus intensity) to stimulate phrenic nerve are given to induce the contraction of the diaphragm to increase the tidal volume. While in the expiratory stage, there is no electrical stimulation rendered to make the diaphragm and expel the gas. Accordingly, the working process of the diaphragm pacemaker is to simulate human physiological respiration. Furthermore, after the operation, the stimulation parameters of the signal in Figure 5 should be adjusted based on the actual situation of the patient.

The waveform of the stimulus affects the physiological response and safety of the nerve. For the safety and simplicity of stimulation, the traditional stimulus waveform adopts square wave in DP [35]. The new stimulus waveforms (see Figure 6) as an physiological reaction of the diaphragm may be more effective, which need further research [36].

To avoid to phrenic nerve injury and diaphragmatic fatigue, patients with SCI to choose appropriate pacing 
TABLE 1: The main difference between three kinds of diaphragm pacemakers $[4,26]$.

\begin{tabular}{lccc}
\hline Manufacturer & Avery, USA & Atrotech, FIN & Medimplant, AUS \\
\hline Developed time & 1960 & 1980 & 1984 \\
Electrodes & Monopolar; bipolar & Quadripolar & Quadripolar \\
Breaths/min & $6-24$ & $8-35$ & $5-60$ \\
Pulse width (ms) & 150 & 200 & $100-1000$ \\
FDA approval & Yes & Invalid & No \\
Electrode & Easy & Relatively difficult & Complicated \\
implantation & Yes & Relatively light & Relatively light \\
Diaphragm fatigue & &
\end{tabular}

TABLE 2: Functional evaluation of the phrenic nerve and diaphragm [31-33].

\begin{tabular}{lrrr}
\hline Method & Implementation process & Evaluation criteria \\
\hline $\begin{array}{l}\text { Nerve conduction } \\
\text { time }\end{array}$ & $\begin{array}{r}\text { Transcutaneous electrical stimulation with a unipolar needle electrode on the neck to } \\
\text { observe the action potential time of diaphragm electromyography }\end{array}$ & $7.5 \sim 9 \mathrm{~ms}[31]$ \\
$\begin{array}{l}\text { X-ray fluoroscopy } \\
\begin{array}{l}\text { Transdiaphragm } \\
\text { pressure }\end{array}\end{array}$ & $\begin{array}{r}\text { Electrical stimulation of phrenic nerve to observe the descending degree of the } \\
\text { diaphragm by X-ray fluoroscopy }\end{array}$ & $>5 \mathrm{~cm}[32](5-8 \mathrm{~cm})$ \\
\hline
\end{tabular}

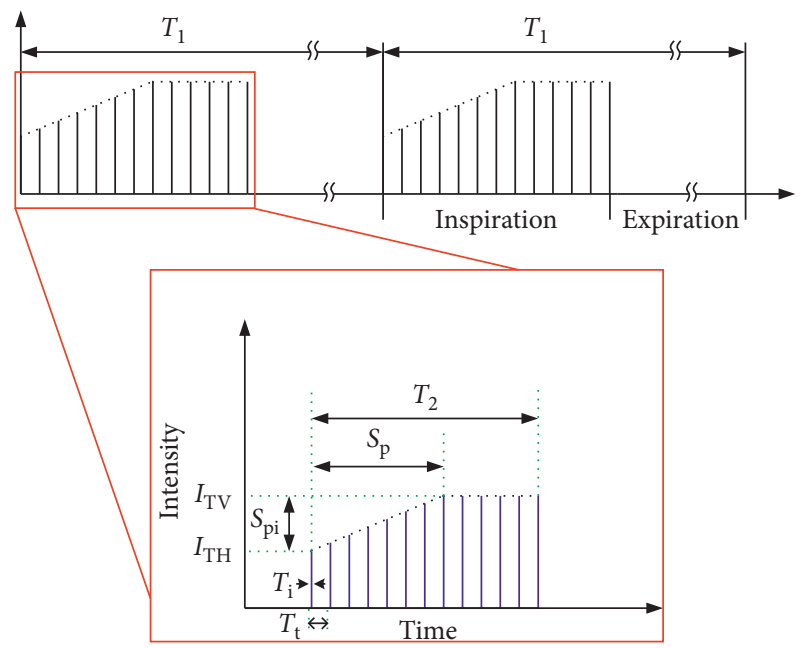

FIGURE 5: Stimulation signal [35]. Stimulation parameters of DP include breathing cycle, stimulation waveform, and pulse width.

parameters is extremely important. Unlike cardiac pacing, a single electrical stimulation pulse does not cause adequate ventilation. DP requires more than 30 continuous stimulation waves at a time. Moreover, several studies have shown that low-frequency pacing and lower respiratory frequency are more tolerable, making muscle structure and function have better chances. Previous successful clinical experience shows that it is safe and effective to adjust the stimulation parameters with pulse frequency of $25 \mathrm{~Hz}$ properly, respiratory frequency of $15 \mathrm{breath} / \mathrm{min}$, and the inspiratory duration of one-third of the respiratory cycle [37] making the diaphragm twitch slowly and fatigue-resistant. In 1981, Oda et al. [38] studied the electrical parameters of DP in dogs. They found that the change of stimulation frequency had a great influence on diaphragmatic fatigue. High frequency $(33 \mathrm{~Hz})$ and narrow pulse interval easily lead to rapid and obvious fatigue. In 2005, Anthony F. DiMarco et al. [39]

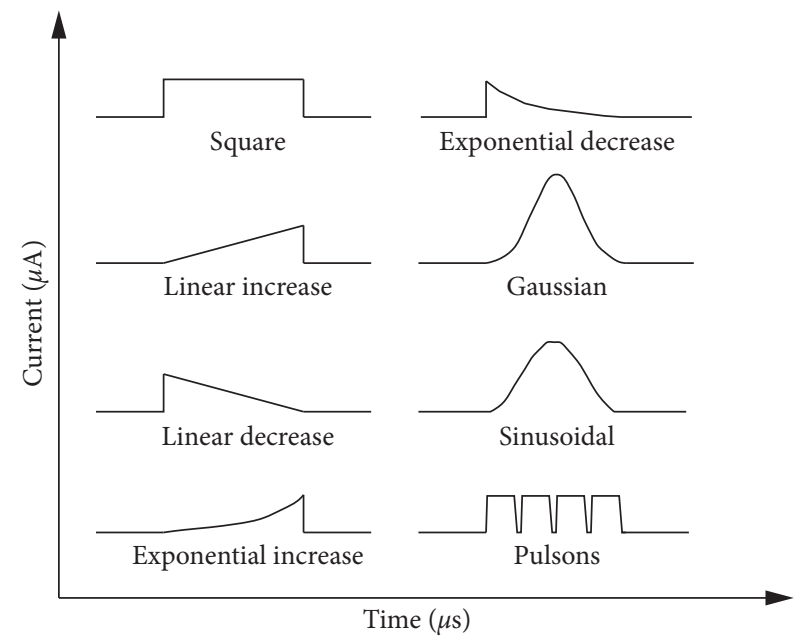

Figure 6: Varied Stimulation waveform [36]. The effects of different stimulus waveforms on neurophysiology need to be further studied.

researched the application of DP in patients with quadriplegia. They set the stimulation parameter (stimulus frequency $20 \mathrm{~Hz}$, inspiratory time 1.1s, and respiratory rate 10-12 breaths/min) to restore the spontaneous breathing in four patients successfully.

However, continuous and long-term stimulation (24 h/ day for several weeks) would harm the function and structure of the diaphragm, resulting in diaphragm fatigue and damage $[40,41]$. The reasonable use time of DP should be in 18 hours/day, so many patients choose to use DP at night and ventilator during the day. Besides, due to the rapid airflow in the trachea caused by DP, the negative pressure in the neck airway may occur, which will give rise to the stenosis of the upper respiratory tract. It was suggested that the continuous use time of DP should not exceed 12 hours or 16 hours a day $[40,41]$. 
As has been mentioned above, interpulse interval, stimulation frequency, and inspiratory time are the key parameters of diaphragm pacemaker. Brouillett [42] discussed the effects of these stimulus parameters on tidal volume for DP in infants and children, as shown in Figures 7 and 8 . Figure 7 shows the trend of tidal volume in three patients at different pulse intervals, which can be fitted into a mathematical formula curve [42]:

$$
V_{T}=\frac{a}{1+\mathrm{be}^{-\mathrm{k} \cdot \mathrm{Hz}}} \text {. }
$$

The value of tidal volume under the stimulation of the shortest (about $30 \mathrm{~ms}$ ) and longest pulse interval approached to two asymptotes.

Similar to Figure 8, the trend of tidal volume value in Figure 8 with the increase of inspiratory time is also by the asymptotic regression, which can be described by [42]

$$
V_{T}=a\left(1-e^{-\mathrm{kt}}\right) \text {. }
$$

Accordingly, based on meeting the needs of patients' ventilation, the balance between less inspiratory time and lower interpulse interval should be achieved in determining pacing parameters.

3.2. Intercostal Muscles Pacing. DP via electric stimulation of bilateral phrenic nerves can restore spontaneous respiratory function in patients with high SCI. However, some patients with SCI are not candidates for diaphragm pacing due to injury of phrenic motor neuron pools or phrenic nerves. Intercostal muscles being the secondary respiratory muscles can contribute $35 \%-40 \%$ of tidal volume. Some researchers have suggested that intercostal muscles can be activated by electrical stimulation [8, 43-46].

A typical study showed that the combined stimulation of the intercostal nerve and unilateral phrenic nerve could separate patients with SCI from the ventilator [44]. This method requires one electrode placed on the ventral surface of the upper thoracic spinal cord by using hemi-laminectomy to activate intercostal muscles and the other electrode implanted into the unilateral phrenic nerve. After 15-30 weeks of recovery after surgery, the patients' inspiratory volume can reach $0.55 \mathrm{~L}-1.31 \mathrm{~L}$, and they can leave the ventilator for spontaneous breathing daily for 16 to 24 hours [44]. Direct stimulation of the upper thoracic spinal cord will cause contraction of the intercostal muscles, triggering the generation of inspired volume. In animal studies, where the diaphragm and intercostal muscles breathe separately, the gas exchange induced by this way alone is the same as that caused by bilateral stimulation of phrenic nerve alone [45].

In addition, high-frequency spinal cord stimulation (HFSCS) is a new and physiologically significant method of activating inspiratory muscles. It was proved that during high-frequency spinal cord stimulation at the T2 level, the peak discharge frequency of a single motor unit of the intercostal muscles was similar to that of spontaneous breathing [46].

Like diaphragmatic pacing, intercostal pacing restores the patients' sense of smell, speech, and social activities and

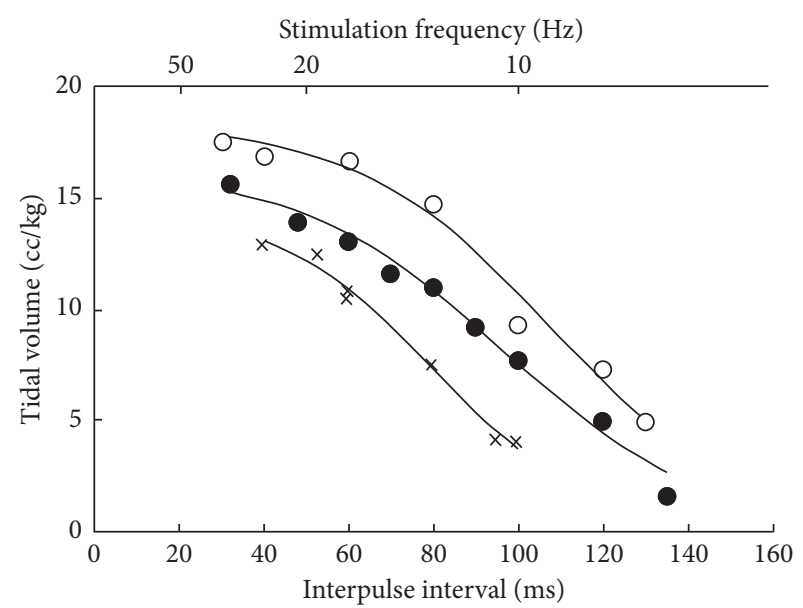

Figure 7: Effect of pulse interval in tidal volume [42]. There are upper and lower limits for the impact of pulse interval on tidal volume.

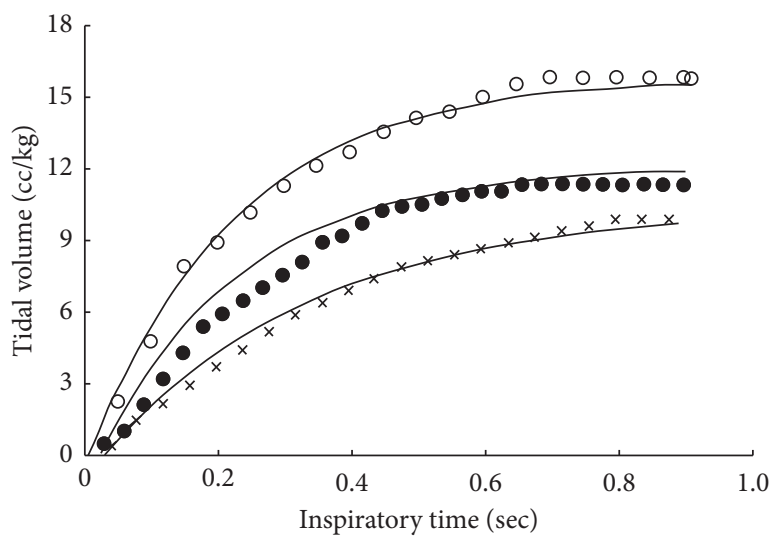

FIGURE 8: Effect of inspiratory time on tidal volume [42]. There is an upper limit to the effect of inspiratory time on tidal volume in stimulation parameters.

generally increases patient happiness. Although this method has some side effects, such as mild hand flexion and upper body muscle contraction, it has less impact on the human body. Intercostal pacing can be a second treatment for patients with unfortunate diaphragm pacing effect. FDA has approved the method through IDE [24], and we believe that it can be applied in the market soon.

\section{Mechanical Ventilation}

4.1. Principle. Mechanical ventilation (MV) with a history of nearly 600 years is one of the essential means for the treatment of respiratory diseases. Since positive-pressure mechanical ventilation (PPMV) successfully rescued polio patients in 1950s, mechanical ventilation support has been widely used in the clinical operation and the treatment of respiratory disorder [47]. As in Figure 9, the ventilator provides positive air pressure higher than the alveolar pressure into the lungs to replace the contraction and expansion of human respiratory muscles $[48,49]$. It can help the patients' respiratory muscles act passively and ensure the 


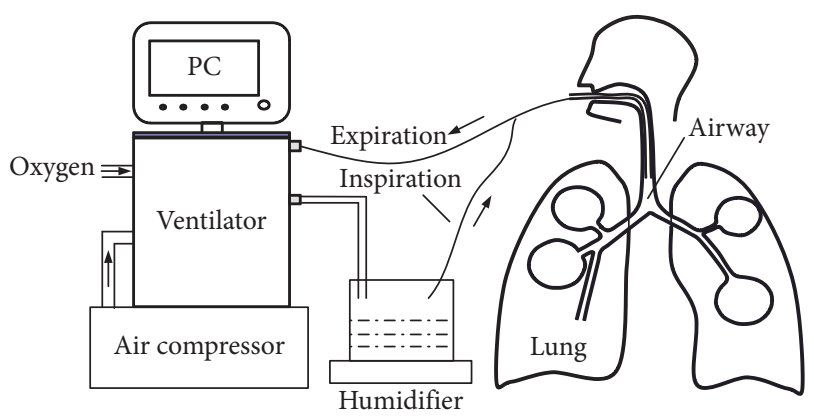

Figure 9: Positive-pressure mechanical ventilation (MV). It is a method to assist or completely replace the patient.

$\mathrm{O}_{2}$ supply and $\mathrm{CO}_{2}$ exhaust. To simulate the function of the human nasal cavity, the gas provided by the ventilator needs to be heated and humidified, and the oxygen concentration is higher than the atmosphere. It is mainly composed of the mechanical module, controller, and human-computer interaction. The mechanical module realises the compression and storage of air and transfers it to the patient [50]. The controller controls the work of the entire ventilator system [50]. Human-computer interaction is mainly for medical staff to set the parameters of the ventilator (tidal volume, pressure, flow, and time) [50].

However, inflating the lung is a nonphysiological ventilation mode, which may contribute to the lung injury characterised pathologically by pneumonedema, oxygen intoxication, and barotraumas [51, 52]. Although the pathogenesis of ventilator-induced lung injury is not fully understood, there is a consensus that the ventilation pressure higher than that of spontaneous respiration will lead to overexpansion of the alveoli and further results in lung injury and the inflammation of body's organs [53, 54]. As a consequence, a concept of pulmonary protective ventilation strategy was proposed to minimise lung injury. Its content mainly includes using low tidal volume to avoid overexpansion of alveoli caused by high tidal volume or airway ventilation, applying sufficient pressure to make the collapsed alveoli open again while inhaling, and keeping the alveoli open with appropriate positive end-expiratory pressure (PEEP) when exhaling [51]. These two ventilation strategies have been proven to be advantageous to prevent alveolar failure and lung injury [55].

4.2. Management of Mechanical Ventilation. According to the connection between the ventilator and the human body, MV can be divided into invasive and noninvasive methods. For the different degrees of respiratory dysfunction caused by SCI, it is necessary for us to accurately evaluate the physiological status of each patient (diaphragmatic function and lung function) and then select the appropriate ventilation mode [56]. The complete SCI patients (C1-C5) with paralysis of the diaphragm and intercostal muscles require to be built effective artificial airway and realisation of MV to ensure the safety of life [57]. For incomplete or low SCI (below C5), conservative treatments can be used. However, the lung function of vital capacity (VC) and arterial blood gas analysis should be strictly monitored to prevent respiratory failure. Clinical practice guidelines for high SCI suggest that patients have an initial tidal volume of $15 \mathrm{ml} / \mathrm{kg}$ for mechanical ventilation and increase with daily chest radiograph atelectasis [58].

Moreover, another critical problem in the management of MV for patients with SCI is the timing of weaning. Whether the patients with SCI can be successfully weaned depends on the location and degree. The success rate of weaning for SCI below $\mathrm{C} 5$ is significantly higher than that above $\mathrm{C} 4$ [59]. The patients' weaning should be performed in strict accordance with the blood gas analysis indexes, leukocyte count, tidal volume, etc. After weaning, the patients' condition should be grimly observed to avoid danger.

\section{Respiratory Muscle Training}

The advancement of the medical level has gradually increased the survival rate of patients with SCI, but the respiratory function of patients with 5 years high SCI will decline [60]. The function of inspiratory and expiratory muscles is inhibited in patients with high SCI due to the injury of neurons controlling the respiratory muscle group. This will lead to the reduction of lung capacity and chest wall compliance, then result in the incidence of atelectasis, dyspnea, and respiratory tract infection, and finally seriously affect the health and quality of life of patients with SCI [61-63]. The degree of respiratory muscle injury depends on the segment and degree of injury.

Respiratory muscle training (RMT), a treatment technique, is to use impedance load, threshold pressure load, and other means to carry out continuous and standardised training of muscles related to respiratory function, which in turn improves the strength and endurance of respiratory muscles [63]. It is inappropriate for such patients with SCI above $\mathrm{C} 3$ segment due to complete paralysis of all respiratory muscles. Patients with SCI (C3-T12 segment) may lead to partial respiratory muscle failure. Therefore, improving the strength of respiratory muscles through RMT for the recovery of respiratory dysfunction due to SCI is effective [63, 64].

RMT, including inspiratory muscle training and expiratory muscle training, needs to choose the appropriate treatment time and intensity. Each treatment should be performed according to the experience of a professional doctor and the guidance of the physiological literature.

SCI patients often use respiratory trainers for respiratory muscle training $[66,67]$. Figure 10 is a photo of expiratory muscle training device [65]. As shown in Figure 11, before using the device, the patient's nose needs to be tightened with a clip [65]. The patient is then asked to take a deep breath, hold the cheeks (to reduce lip leaks), and blow hard on the device. When the device detects that the air pressure reaches a threshold, they are reminded to stop exhaling. The treatment requires to take approximately $4-8$ weeks, and approximately 25 to 30 training sessions need to be completed each day [68-70]. Clinicians can check the maximum expiratory pressure (MEP) and pulmonary function parameters (FEV1 and FVC) of trained patients every week to evaluate the effect of treatment. 


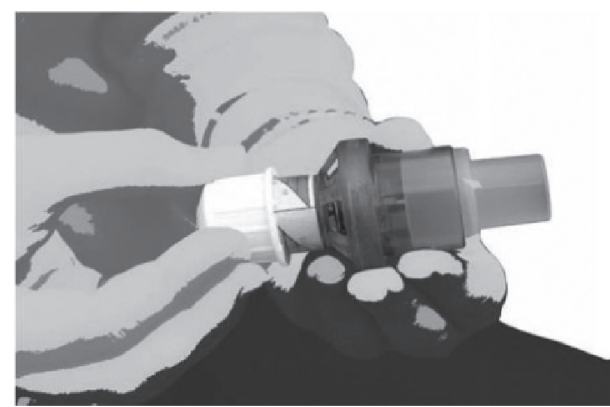

Figure 10: Expiratory muscle trainer [65]. It sets a pressure threshold through a spring-controlled check valve to enhance expiratory muscle strength.

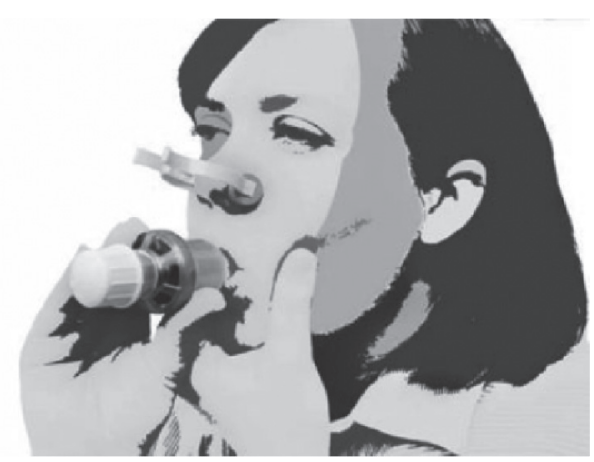

FIGURE 11: Respiratory muscle training posture [65]. The patient holds the device with his lips, bites his mouth, and tries to blow into the device.

Many clinicians have studied the effect of RMT on the recovery of respiratory function in patients with SCI. There are two main methods they use to study this subject: one is clinical research verification and the other is meta-analysis or review based on literature search. Postma concluded that, after the treatment of inspiratory muscle impedance, the maximum inspiratory pressure and mental state of 40 patients with SCI had a short-term improvement [64]. Rutchik et al. conducted respiratory muscle confrontation training for 10 patients with cervical spinal cord injury for 8 weeks. Then, they calculated that these patients' mechanical parameters such as vital capacity, maximum inspiratory pressure, and functional residual capacity all increased [71]. Also, meta-analysis results showed that RMT is more effective than traditional rehabilitation methods in improving vital capacity, inspiratory capacity, maximum inspiratory pressure, and maximum voluntary ventilation [63]. In general, RMT can effectively reduce the probability of lung infection in patients with SCI and improve lung function. Therefore, RMT is worth promoting in the clinic.

\section{Discussion}

Currently, various techniques of respiratory function reconstruction of SCI have been widely used in clinical practice, effectively maintaining the lives of some patients. However, the shortcomings and limitations of these technologies have led to many patients not being able to get treatment or poor treatment results.

MV is the preferred method of restoring respiratory function in patients with SCI. It can provide temporary respiratory support and continue the patients' life. However, this is a way to help patients breathe passively and cannot help patients with spontaneous breathing function. At the same time, prolonged mechanical ventilation can cause problems such as bedsores, respiratory infections, and airway secretion clearance problem [72]. Compared to MV, DP can restore the patients' language function and social ability and reduce the economic burden [73, 74]. The cost of performing diaphragmatic pacing is expensive. The price of each type of DP is between US\$50000-60000 [4]. Surgery, hospitalisation, and follow-up costs add another US $\$ 20000$ [4]. Therefore, DP is more likely to be accepted by patients with SCI. The prerequisite for breathing muscle training is that the patient has a certain ability to breathe spontaneously, which causes that respiratory muscle training can only be used as an auxiliary respiratory function rehabilitation treatment for patients with mild SCI.

Overall, the recovery of respiratory function for SCI is developing in a direction that is meeting the physiological needs of patients. DP is the most effective in improving participants' quality of life and happiness among the three treatments. However, some unavoidable fatal disadvantages of DP, such as equipment failure, postoperative infection, and unknown neurological damage, need to be urgently addressed [24].

The development of microelectronic technology has promoted the improvement of the control function and expansion function of the single-chip microcomputer, which has also been widely used in medical instruments and equipment. Meanwhile, modern control theory achieves fast response and stability of complex systems with time-varying and multivariable [75-78]. The medical hardware platform based on single-chip technology can accurately process and recognize the biological signals of the human body in real time $[79,80]$. Zbrzeski developed a multifunctional hardware platform to realise the real-time collection, calculation, display, and storage of biological signals and then applied it to the animal model of DP.

For the current open-loop DP system not responding to changes in equipment components and physiological needs, some researchers [81, 82] have proposed to establish a closed-loop diaphragm pacing system relying on changes in respiratory airflow (Figure 12). Nevertheless, closed-loop DP has not yet appeared on the market and in clinical applications.

The PG/PS controller [81, 83], containing two modules PG and PS, builds a closed-loop diaphragm pacing system. The pacing cycle is set in the PG module, and the PS module adaptively adjusts the stimulation parameters with the change of actual respiratory airflow to make ideal respiratory airflow. The PS module consists of a single-layer neural network, in which each neuron outputs a raised cosine function $\left(y_{j}(t)\right)$ as a basic function. The output value $Z(t)$ of PS is the sum of the weighted values $\left(\omega_{j}(t)\right)$ of the basic functions: 


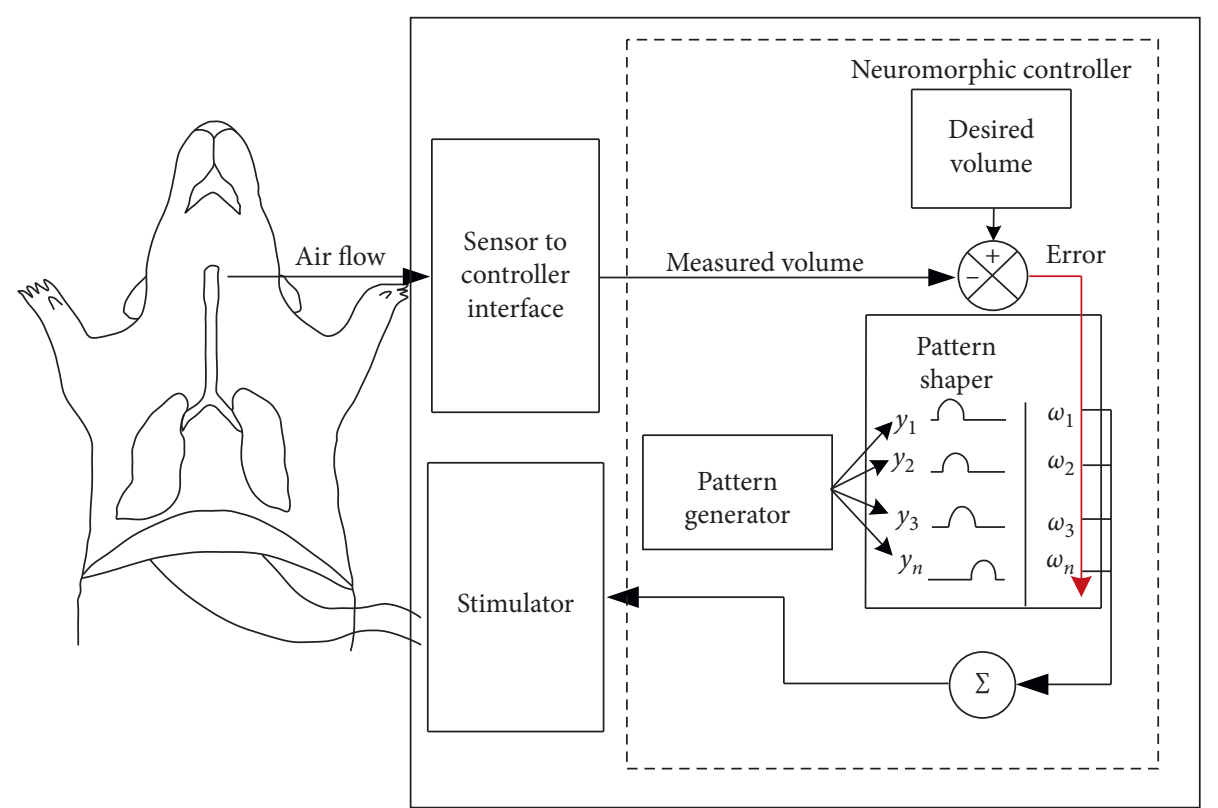

FIGURE 12: Animal model of closed-loop diaphragm pacing system [81]. The system controller adjusts the stimulation parameters online based on the error between the measured respiratory airflow and the ideal airflow.

$$
Z(t)=\sum_{j=1}^{m} \omega_{j}(t) y_{j}(t) .
$$

Also, the EMG signal reflects muscle movement [84] and the respiratory parameters can also be evaluated through the characteristics of the diaphragm EMG signal [85, 86]. Therefore, using the surface diaphragmatic myoelectric signal as feedback is also a research direction to establish a closed-loop diaphragmatic pacing system.

\section{Conclusions}

High spinal cord injury is one of the most severe injuries, which can lead to death from respiratory failure. This review focuses on respiratory dysfunction in patients with SCI and uses three commonly used devices to restore respiratory function, namely, electrical stimulation, mechanical ventilation, and respiratory muscle training. Compared with the other two treatment methods, electrical stimulation is the most effective treatment method.

Improvements in current respiratory therapy for SCI should underline safety, reliability, and intelligence, which means close to human physiology, fewer complications and side effects, and reliable product performance. Real prospects are presented below:

(1) Investigate neural signals more meet respiratory physiological patterns. When the pulse signal stimulates the phrenic nerve, the patient produces a rapid ventilation. Hence, a stimulus waveform has the same volume of ventilation as a person's spontaneous breathing should be explored.

(2) Improve diaphragmatic pacemaker surgery. Highfrequency spinal cord electrical stimulation is also the development direction of diaphragm pacing in the future due to the advantages of less surgical trauma and closer to the physiological mode.

(3) Emphasize joint stimulation between the diaphragm and other respiratory muscles to make the respiratory airflow closer to the normal respiratory airflow.

\section{Conflicts of Interest}

The authors declare that they have no conflicts of interest.

\section{Acknowledgments}

The research was funded by Grant (2019M660391) of China Postdoctoral Science Foundation and the Open Foundation of the State Key Laboratory of Fluid Power and Mechatronic Systems (GZKF-201920).

\section{References}

[1] R. Kumar, J. Lim, R. A. Mekary et al., "Traumatic spinal injury: global epidemiology and worldwide volume," World Neurosurgery, vol. 113, pp. 345-363, 2018.

[2] Y. S. Xu, D. Q. Luo, W. H. Pan et al., "Epidemiological analysis of traumatic cervical spinal cord injury," Chinese Journal of Emergency Medicine, vol. 28, no. 1, pp. 84-89, 2019.

[3] R. Brown, A. F. DiMarco, J. D. Hoit, and E Garshick, "Respiratory dysfunction and management in spinal cord injury," Respiratory Care, vol. 51, no. 8, pp. 853-870, 2006.

[4] A. F. DiMarco and G. H. Creasey, "Neuroprosthetic control of respiratory muscles," Series on Bioengineering and Biomedical Engineering, vol. 40, no. 12, pp. 566-600, 2017.

[5] A. Alshekhlee, R. P. Onders, T. U. Syed, M. Elmo, and B. Katirji, "Phrenic nerve conduction studies in spinal cord injury: applications for diaphragmatic pacing," Muscle \& Nerve, vol. 38, no. 6, pp. 1546-1552, 2008. 
[6] A. F. DiMarco and K. E. Kowalski, "Effects of chronic electrical stimulation on paralyzed expiratory muscles," Journal of Applied Physiology, vol. 104, no. 6, pp. 1634-1640, 2008.

[7] A. F. Dimarco, "Neural prostheses in the respiratory system," Journal of Rehabilitation Research and Development, vol. 38, no. 6, pp. 601-607, 2001.

[8] A. F. DiMarco, M. D. Altose, A. Cropp et al., "Activation of the Inspiratory intercostal muscles by electrical stimulation of the spinal cord," American Review Respiratory Disease, vol. 136, no. 6, pp. 1385-1390, 1988.

[9] L. Guth, L. Soutter, K. Frank, J. B. Campbell, and J. B. Lloyd, "Diaphragmatic function following anastomosis of recurrent laryngeal and phrenic nerves," Experimental Neurology, vol. 2, no. 3, pp. 251-260, 1960.

[10] J. Polentes, J. C. Stamegna, M. Nieto-Sampedro, and P. Gauthier, "Phrenic rehabilitation and diaphragm recovery after cervical injury and transplantation of olfactory ensheathing cells," Neurobiology of Disease, vol. 16, no. 3, pp. 638-653, 2004.

[11] N. J. Livingston, "The intercostal to phrenic nerve transfer: an effective means of reanimating the diaphragm in patients with high cervical spine injury," Plastic and Reconstructive Surgery, vol. 105, no. 4, pp. 1255-1261, 2000.

[12] National Spinal Cord Injury Statistical Center, "Spinal cord injury facts and figures at a glance," The Journal of Spinal Cord Medicine, vol. 28, no. 4, pp. 379-380, 2005.

[13] World Health Organization and International Spinal Cord Society, International Perspectives on Spinal Cord Injury, pp. 3-10, World Health Organization, Geneva, Switzerland, 2013.

[14] Y. J. HAN, G. M. LI, D. H. LUO et al., "The influencing factors of early death in cervical spinal cord injury patients (A report of 81 cases)," China Medical Herald, vol. 5, no. 18, pp. 42-43, 2008.

[15] A. B. Jackson and T. E. Groomes, "Incidence of respiratory complications following spinal cord injury," Archives of Physical Medicine and Rehabilitation, vol. 75, no. 3, pp. 270-275, 1994.

[16] R. P. Onders, "Functional electrical stimulation," Handbook of Clinical Neurology, vol. 109, pp. 275-282, 2012.

[17] W. W. Glenn, J. H. Hageman, A. Mauro et al., "Electrical stimulation of excitable tissue by radio frequency transmission," Annals of Surgery, vol. 160, no. 3, pp. 338-350, 1964.

[18] W. W. L. Glenn, W. G. Holcomb, A. J. McLaughlin, J. M. O’Hare, J. F. Hogan, and R. Yasuda, “Total ventilatory support in a quadriplegic patient with radiofrequency electrophrenic respiration," New England Journal of Medicine, vol. 286, no. 10, pp. 513-516, 1972.

[19] W. W. Glenn and M. L. Phelps, "Diaphragm pacing by electrical stimulation of the phrenic nerve," Neurosurgery, vol. 17, no. 6, pp. 974-984, 1985.

[20] P. Khong, A. Lazzaro, and R. Mobbs, "Phrenic nerve stimulation: the Australian experience," Journal of Clinical Neuroscience, vol. 17, no. 2, pp. 205-208, 2010.

[21] J. A. Elefteriades, J. A. Quin, J. F. Hogan et al., "Long-term follow-up of pacing of the conditioned diaphragm in quadriplegia," Pacing and Clinical Electrophysiology, vol. 25, no. 6, pp. 897-906, 2002.

[22] W. W. L. Glenn, J. F. Hogan, M. L. Phelps et al., "Ventilatory support of the quadriplegic patient with respiratory paralysis by diaphragm pacing," Surgical Clinics of North America, vol. 60 , no. 5, pp. 1055-1078, 1980.

[23] W. W. L. Glenn, J. F. Hogan, J. S. O. Loke, T. E. Ciesielski, M. L. Phelps, and R. Rowedder, "Ventilatory support by pacing of the conditioned diaphragm in quadriplegia," New England Journal of Medicine, vol. 310, no. 18, pp. 1150-1155, 1984.

[24] A. F. DiMarco, "Phrenic nerve stimulation in patients with spinal cord injury," Respiratory Physiology \& Neurobiology, vol. 169, no. 2, pp. 200-209, 2009.

[25] S. V. Abdunnur and D. H. Kim, "Phrenic nerve stimulation: technology and clinical applications," Stimulation of the Peripheral Nervous System, vol. 29, pp. 64-75, 2016.

[26] G. Creasey, J. Elefteriades, A. DiMarco et al., "Electrical stimulation to restore respiration," Journal of Rehabilitation Research and Development, vol. 33, no. 2, pp. 123-132, 1996.

[27] W. T. Abraham, D. Jagielski, O. Oldenburg et al., "Phrenic nerve stimulation for the treatment of central sleep apnea," JACC: Heart Failure, vol. 3, no. 5, pp. 360-369, 2015.

[28] M. L. Yang, H. M. Zhao, J. J. Li et al., "The clinic experience of implantable diaphragm pacer in a patient with high cervical spinal cord injury and literature review," Chinese Journal of Tuberculosis and Respiratory Diseases, vol. 41, no. 9, pp. 718-723, 2018.

[29] R. Chen, L. I. Jianjun, X. Meng et al., "Application of diaphragm pacing technology in respiratory function reconstruction in patients with cervical spinal cord injury," Chinese Journal of Rehabilitation Theory and Practice, vol. 21, no. 2, pp. 157-162, 2015.

[30] O. Oldenburg, T. Bitter, H. Fox, D. Horstkotte, and K.-J. Gutleben, "Effects of unilateral phrenic nerve stimulation on tidal volume," Herz, vol. 39, no. 1, pp. 84-86, 2014.

[31] D. K. McKenzie and S. C. Gandevia, "Phrenic nerve conduction times and twitch pressures of the human diaphragm," Journal of Applied Physiology, vol. 58, no. 5, pp. 1496-1504, 1985.

[32] R. K. Shaw, W. W. L. Glenn, J. F. Hogan, and M. L. Phelps, "Electrophysiological evaluation of phrenic nerve function in candidates for diaphragm pacing," Journal of Neurosurgery, vol. 53, no. 3, pp. 345-354, 1980.

[33] A. Mier, C. Brophy, J. Moxham, and M. Green, "Twitch pressures in the assessment of diaphragm weakness," Thorax, vol. 44, no. 12, pp. 990-996, 1989.

[34] A. K. Karlsson, L. Dernevik, and B. Houltz, "Spinal cord injuries. An intact nerve can be enough for a successful phrenic nerve stimulation," Lakartidningen, vol. 106, no. 11, pp. 11-17, 2009.

[35] X. Lu, D. Guiraud, S. Renaux, T. Similowski, and C. A. Coste, Monitoring Phrenic Nerve Stimulation-Induced Breathing via Tracheal Sounds, Hal-Lirmm, RehabWeek, Toronto, Canada, 2019.

[36] C. Günter, J. Delbeke, and M. Ortiz-Catalan, "Safety of longterm electrical peripheral nerve stimulation: review of the state of the art," Journal of Neuro Engineering and Rehabilitation, vol. 16, no. 13, pp. 1-16, 2019.

[37] W. W. L. Glenn, W. G. Holcomb, J. Hogan et al., "Diaphragm pacing by radiofrequency transmission in the treatment of chronic ventilatory insufficiency," The Journal of Thoracic and Cardiovascular Surgery, vol. 66, no. 4, pp. 505-520, 1973.

[38] T. Oda, W. W. L. Glenn, Y. Fukuda, J. F. Hogan, and J. Gorfien, "Evaluation of electrical parameters for diaphragm pacing: an experimental study," Journal of Surgical Research, vol. 30, no. 2, pp. 142-153, 1981.

[39] A. F. DiMarco, R. P. Onders, A. Ignagni, K. E. Kowalski, and J. T. Mortimer, "Phrenic nerve pacing via intramuscular diaphragm electrodes in tetraplegic subjects," Chest, vol. 127, no. 2, pp. 671-678, 2005. 
[40] W. W. L. GLENN, “Diaphragm pacing: present status,” Pacing and Clinical Electrophysiology, vol. 1, no. 3, pp. 357-370, 1978.

[41] C. E. Hunt, S. V. Matalon, T. R. Thompson et al., "Central hypoventilation syndrome: experience with bilateral phrenic nerve pacing in 3 neonates," American Review of Respiratory Disease, vol. 118, no. 1, pp. 23-28, 1978.

[42] R. T. Brouillette, M. N. Ilbawi, L. Klemka-Walden, and C. E. Hunt, "Stimulus parameters for phrenic nerve pacing in infants and children," Pediatric Pulmonology, vol. 4, no. 1, pp. 33-38, 1988.

[43] A. F. DiMarco, K. Budzinska, and G. S Supinski, "Artificial ventilation by means of electrical activation of intercostal/ accessory muscles alone in anesthetized dogs," American Review of Respiratory Disease, vol. 139, no. 4, pp. 961-967, 1988.

[44] A. F. DiMarco, Y. Takaoka, and K. E. Kowalski, "Combined intercostal and diaphragm pacing to provide artificial ventilation in patients with tetraplegia," Archives of Physical Medicine and Rehabilitation, vol. 86, no. 6, pp. 1200-1207, 2005.

[45] A. F. DiMarco, A. F. Connors Jr, and K. E. Kowalski, "Gas exchange during separate diaphragm and intercostal muscle breathing," Journal of Applied Physiology, vol. 96, no. 6, pp. 2120-2124, 2004.

[46] A. F. DiMarco and K. E. Kowalski, "Intercostal muscle pacing with high frequency spinal cord stimulation in dogs," Respiratory Physiology \& Neurobiology, vol. 171, no. 3, pp. 218-224, 2010.

[47] B. Ibsen, “The anaesthetist's viewpoint on the treatment of respiratory complications in poliomyelitis during the epidemic in Copenhagen, 1952," Proceedings of the Royal Society of Medicine, vol. 47, no. 1, pp. 72-74, 1954.

[48] E. Kondili, G. Prinianakis, and D. Georgopoulos, "Patientventilator interaction," British Journal of Anaesthesia, vol. 91, no. 1, pp. 106-119, 2003.

[49] S. Ren, M. L. Cai, Y. Shi, W. Q. Xu et al., "Influence of bronchial diameter change on the airflow dynamics based on a pressure-controlled ventilation system," International Journal for Numerical Methods in Biomedical Engineering, vol. 34, no. 3, pp. 1-14, 2018.

[50] F. Zhou, W. Guan, K. Sierszecki et al., "Component-based design of software for embedded control systems: the medical ventilator case study," in Proceedings of the 2009 International Conference on Embedded Software and Systems, pp. 157-163, IEEE, Zhejiang, China, May 2009.

[51] A. S. Slutsky and V. M Ranieri, "Ventilator-induced lung injury," The New England Journal of Medicine, vol. 369, pp. 2216-2136, 2013.

[52] G. Nash, J. B. Blennerhassett, and H. Pontoppidan, "Pulmonary lesions associated with oxygen therapy and artificial ventilation," New England Journal of Medicine, vol. 276, no. 7, pp. 368-374, 1967.

[53] R. D. Stevens, C. Lazaridis, and J. A. Chalela, "The role of mechanical ventilation in acute brain injury," Neurologic Clinics, vol. 26, no. 2, pp. 543-563, 2008.

[54] M. Kearns and D. Shimabukuro, "Respiratory complications and management of mechanical ventilation in cervical spine injury," ICU Director, vol. 3, no. 5, pp. 220-223, 2012.

[55] M. B. P. Amato, C. S. V. Barbas, D. M. Medeiros et al., "Effect of a protective-ventilation strategy on mortality in the acute respiratory distress syndrome," New England Journal of Medicine, vol. 338, no. 6, pp. 337-354, 1998.

[56] R. Galeiras Vázquez, P. Rascado Sedes, M. Mourelo Fariña, A. Montoto Marqués, and M. E. Ferreiro Velasco,
"Respiratory management in the patient with spinal cord injury," BioMed Research International, vol. 2013, Article ID 168757, 12 pages, 2013.

[57] E. B. Marsolais, M. L. Boninger, P. McCormick et al., "Respiratory management following spinal cord injury: a clinical practice guideline for health care professionals," Journal of Spinal Cord Medicine, vol. 28, no. 3, pp. 259-293, 2005.

[58] S. Berney, P. Bragge, C. Granger, H. Opdam, and L. Denehy, "The acute respiratory management of cervical spinal cord injury in the first 6 weeks after injury: a systematic review," Spinal Cord, vol. 49, no. 1, pp. 17-29, 2011.

[59] A. E. Chiodo, W. Scelza, and M. Forchheimer, "Predictors of ventilator weaning in individuals with high cervical spinal cord injury," The Journal of Spinal Cord Medicine, vol. 31, no. 1, pp. 72-77, 2008.

[60] K. Postma, J. A. Haisma, S. de Groot et al., "Changes in pulmonary function during the early years after inpatient rehabilitation in persons with spinal cord injury: a prospective cohort study," Archives of Physical Medicine and Rehabilitation, vol. 94, no. 8, pp. 1540-1546, 2013.

[61] C. P. Cardozo, "Respiratory complications of spinal cord injury," The Journal of Spinal Cord Medicine, vol. 30, no. 4, pp. 307-308, 2007.

[62] R. Brown, A. F. DiMarco, J. D. Hoit et al., "Respiratory dysfunction and management in spinal cord injury," Respiratory Care, vol. 51, no. 8, pp. 869-870, 2006.

[63] Y. Xu, Y. Gao, Q. Xie et al., "Efficacy of respiratory muscle training on pulmonary function of spinal cord injury patients: a meta-analysis," Chinese Journal Of Evidence-Based Medicine, vol. 17, no. 10, pp. 1150-1157, 2017.

[64] K. Postma, J. A. Haisma, M. T. E. Hopman, M. P. Bergen, H. J. Stam, and J. B. Bussmann, "Resistive inspiratory muscle training in people with spinal cord injury during inpatient rehabilitation: a randomized controlled trial," Physical Therapy, vol. 94, no. 12, pp. 1709-1719, 2014.

[65] C. Sapienza, M. Troche, T. Pitts, and P. Davenport, "Respiratory strength training: concept and intervention outcomes," Seminars in Speech and Language, vol. 32, no. 1, pp. 021-030, 2011.

[66] E. J. Roth, K. W. Stenson, S. Powley et al., "Expiratory muscle training in spinal cord injury: a randomized controlled trial," Archives of Physical Medicine and Rehabilitation, vol. 91, no. 6, pp. 857-861, 2010.

[67] Y. Bao and J. H. Sun, "The effect of respiratory trainer in the care of the patients with cervical spinal cord injuries," Medical Journal of West China, vol. 23, no. 12, pp. 2449-2450, 2011.

[68] A. Schlumberger, J. Stec, D. Schmidtbleicher et al., "Single- vs. multiple-set strength training in women," The Journal of Strength and Conditioning Research, vol. 15, no. 3, pp. 284289, 2001.

[69] R. M. Otto and R. N. Carpinelli, "A critical analysis of the single versus multiple set debate," Journal of Exercise Physiology Online, vol. 9, no. 1, pp. 32-57, 2006.

[70] M. R. Rhea, B. A. Alvar, and L. N. Burkett, "Single versus multiple sets for strength: a meta-analysis to address the controversy," Research Quarterly for Exercise and Sport, vol. 73, no. 4, pp. 485-488, 2002.

[71] A. Rutchik, A. R. Weissman, P. L. Almenoff, A. M. Spungen, W. A. Bauman, and D. R. Grimm, "Resistive inspiratory muscle training in subjects with chronic cervical spinal cord injury," Archives of Physical Medicine and Rehabilitation, vol. 79, no. 3, pp. 293-297, 1998.

[72] S. Ren, Y. Shi, M. L. Cai et al., "Influence of airway secretion on airflow dynamics of mechanical ventilated respiratory 
system," IEEE/ACM Transactions on Computational Biology and Bioinformatics, vol. 15, no. 5, pp. 1660-1668, 2017.

[73] W. H. Dobelle, M. S. D’Angelo, B. F. Goetz et al., "200 cases with a new breathing pacemaker dispel myths about diaphragm pacing," ASAIO Journal, vol. 40, no. 3, pp. 244-252, 1994.

[74] M. N. Ilbawi, F. S. Idriss, C. E. Hunt et al., "Diaphragmatic pacing in infants. Techniques and results," The Annals of Thoracic Surgery, vol. 21, no. 11, pp. 323-329, 1985.

[75] Z. Li, W. Zuo, and S. Li, "Zeroing dynamics method for motion control of industrial upper-limb exoskeleton system with minimal potential energy modulation," vol. 163, pp. 110, 2020.

[76] Z. Li, C. Li, S. Li et al., "A fault-tolerant method for motion planning of industrial redundant manipulator," IEEE Transactions on Industrial Informatics, vol. 16, 2019.

[77] H. Su, W. Qi, C. Yang, J. Sandoval, G. Ferrigno, and E. D. Momi, "Deep neural network approach in robot tool dynamics identification for bilateral teleoperation," IEEE Robotics and Automation Letters, vol. 5, no. 2, pp. 2943-2949, 2020.

[78] W. Qi, H. Su, and A. Aliverti, “A smartphone-based adaptive recognition and real-time monitoring system for human activities," IEEE Transactions on Human-Machine Systems, 2020.

[79] A. Quotb, J. B. Floderer, T. Bollengier et al., "Live demonstration: real-time wavelet spike detection with in-vitro biological signals," in Proceedings of the 2012 IEEE Biomedical Circuits and Systems Conference (BioCAS), p. 79, Hsinchu, Taiwan, November 2012.

[80] A. M. Zbrzeski, R. Siu, Y. Bornat et al., "A versatile fastdevelopment platform applied to closed-loop diaphragmatic pacing," in Proceedings of the 2015 7th International IEEE/ EMBS Conference on Neural Engineering (NER), pp. 791-794, Montpellier, France, April 2015.

[81] R. Siu, J. J. Abbas, B. K. Hillen et al., "Restoring ventilatory control using an adaptive bioelectronic system," Journal of Neurotrauma, vol. 36, no. 24, pp. 3363-3377, 2019.

[82] B. K. Hillen, J. J. Abbas, A. Zbrzeski et al., "Adaptive control of ventilation usingelectrical stimulation in a biomechanical model," BMC Neuroscience, vol. 16, no. 1, pp. 1-2, 2015.

[83] M. D. Fairchild, S.-J. Kim, A. Iarkov, J. J. Abbas, and R. Jung, "Repetetive hindlimb movement using intermittent adaptive neuromuscular electrical stimulation in an incomplete spinal cord injury rodent model," Experimental Neurology, vol. 223, no. 2, pp. 623-633, 2010.

[84] H. Su, S. E. Ovur, X. Zhou et al., "Depth vision guided hand gesture recognition using electromyographic signals," Advanced Robotics, vol. 15, pp. 1-13, 2020.

[85] L. Estrada, A. Torres, L. Sarlabous et al., "Improvement in neural respiratory drive estimation from diaphragm electromyographic signals using fixed sample entropy," IEEE Journal of Biomedical and Health Informatics, vol. 20, no. 2, pp. 476-485, 2015.

[86] L. Estrada, A. Torres, L. Sarlabous et al., "Onset and offset estimation of the neural inspiratory time in surface diaphragm electromyography: a pilot study in healthy subjects," IEEE Journal of Biomedical and Health Informatics, vol. 22, no. 1, pp. 67-76, 2017. 